\title{
Transportation Backhaul Matching Using Binary Programming Model: A Case Study on Third-Party Logistics Network in Thailand
}

\author{
Machigar Ongtang and Satama Sirivunnabood
}

\begin{abstract}
Backhauling has become an attractive cost-saving approach in logistics. To explore such opportunities, we propose a backhaul matching optimization model based on Binary Integer Programming (BIP). A set of operational and logical constraints was also developed. While flexible enough to apply to general logistics networks, we validate our model in a large 3rd party logistics network in Thailand. It is shown that our scheme always produces optimal backhaul matching plan, satisfies the defined constraints, and in turn outperforms the existing manual operation that is also time-consuming and subject to human error. The proposed model provides a promising scheme to improve the network's backhaul matching operation and yield a competitive advantage.
\end{abstract}

Index Terms-Backhaul matching, binary integer programming, logistics, supply chain network.

\section{INTRODUCTION}

Transportation cost is usually counted as a significant part of total logistics cost. This is particularly true for the products with relatively low production cost compared to its total manufacturing cost, such as agricultural, traditional construction materials, and other commodity products. For instance, the recent logistics survey [1] shows that the logistics cost is approximately $7.87 \%$ of the total sales for average companies, $44 \%$ of which is the transportation cost. For Thailand, the logistics cost as a percentage of sales is more than $20 \%$ on average [2]; $47.2 \%$ of this proportion contributes to the transportation cost. Apparently, efforts have been spent in order to improve this non-ignorable cost, including truck fleet sharing, backhaul matching, shipment consolidation, etc. In this paper, we emphasize our discussion on a cost improvement strategy through truck backhaul matching, which can be accomplished by identifying the most appropriate backhaul shipment to a particular linehaul, in order to reduce the number of trucks travelling with empty load, and in turn improve the transportation cost. This cost-saving practice usually provides the greatest benefits when manufacturing firms outsource their transportation operations to 3rd Party Logistics (3PL) Companies. This beneficial condition results from a broad variety of linehaul and backhaul products, which the 3PL company can optimally select from its customer portfolios. We propose a Binary Integer

Manuscript received January 5, 2014; revised March 14, 2014.

Machigar Ongtang is with Dhurakij Pundit University, Bangkok 10210 , Thailand (e-mail: machigar.ong@dpu.ac.th).

Satama Sirivunnabood is with King Mongkut's Institute of Technology Ladkrabang, Bangkok 10520, Thailand (e-mail: satamas@gmail.com).
Programming (BIP) model to identify an optimal backhaul matching plan with minimal number of empty load travelling.

This paper is organized into seven sections. Some related literatures are discussed in Section II. Section III describes our problem scenario in detail. Section IVdemonstrates the development of our proposed BIP model for the backhaul matching problem. Section $\mathrm{V}$ outlines our experiment in a 3PL network focusing on commodity products in Thailand. The meaningful results along with some discussion are presented in Section VI. Lastly, Section VII draws this paper to conclusion.

\section{LITERATURE REVIEW}

Previous contributions in the area of backhaul analysis and optimization models mostly examine vehicle routing problem with backhauls (VRPB) [3], which is a class of pick-up/delivery problem. It considers the delivery of goods from a depot to a number of delivery points. On each route, after all deliveries are made, returnable items are picked up and brought back to the depot. VRPB is generally an NP-hard combinatorial optimization problem. Therefore, this paradigm usually adopts heuristic approaches such as tabu search [4], [5], genetic algorithms [6], ant colony optimizations [7], and local-search metaheuristic method [8], to obtain approximate solutions in acceptable time.

Another class of backhaul study involves flow planning instead of operational routing problem. Carlsson et al. [9] presented a backhaul flow planning model in forestry. Their decision variables identify flows between a set of supply points and linehaul demand points with additional flow variables representing potential backhaul routes. The model is formulated as a linear programming problem, whereas a solution method based on column generation is used to guarantee an optimal solution. In contrast, we focus on backhaul matching plan, which considers optimal backhaul matches for a set of linehaul destination points and backhaul supply points. Moreover, the transported volume per truck is not our concern as our scenario always involves full truckload of nearly steady volume. Consequently, binary programming is more appropriate for our settings.

In spite of backhaul optimization, data mining technique was also introduced to this area [10], [11]. Muckell et al. [11] proposed an intelligent brokerage system, which automatically identifies backhaul and load sharing opportunities. It utilizes telematics data such as sensor and geo-based information to create historical pattern of freight movement and detect backhaul opportunities. 


\section{PROBLEM DESCRIPTION}

Let us consider a generalized logistics network with backhaul matching opportunity operated by a 3PL company as illustrated in Fig. 1. The network includes Nlinehaul source nodes, denoted as $l s r c_{1}, l s r c_{2}, \ldots l s r c_{N}$, where $N$ is a large integer. For each route $i$, linehaul products are shipped from $l s r c_{i}$ to a linehaul destination or sink node, $l s n k_{i}$, on day $t$. Then after the delivery, the truck will travel with empty load to pick up a backhaul product at a backhaul source node, indicated as $b s r c_{j}$, which is in an area close to $l s n k_{i}$. The backhaul shipment must be collected on or within day $t+d$, where the value of $d$ depends on the 3PL company's logistics planning policy. Once the truck is loaded with the backhaul product, it travels back to an area near by $l s r c_{i}$ to deliver the backhaul at the backhaul destination or sink node, denoted as $b s n k_{j}$, which maybe also be $l s r c_{i}$.

It can be realized from this scenario that there are several possible combinations when developing a complete backhaul matching plan, which aims to match linehaul shipments to appropriate backhaul shipments. However, our objective is to develop the backhaul matching plan such that the number of trucks with empty load travelling back to the linehaul source nodes is minimized (i.e. maximize the number of matchings). Additionally, four main conditions must be kept in mind. First, the distance between the linehaul sink node and backhaul source node, also known as "deadhead," must not exceed a predetermined value. Second, the delay time that the truck needs to wait before loading the backhaul product shall be no longer than specified. Third, the products of the linehaul and backhaul shipments shall not be in the categories that contaminated each other. Lastly, the truck types, i.e. flatbed trailers or dump trucks, required by the line hauland backhaul shipments to be matched must be the same. The expected result of this modeling task will be the backhaul matching plan identifying which possible pairs of linehaul and backhaul shipments should be matched in order to maximize the number of backhaul matchings.

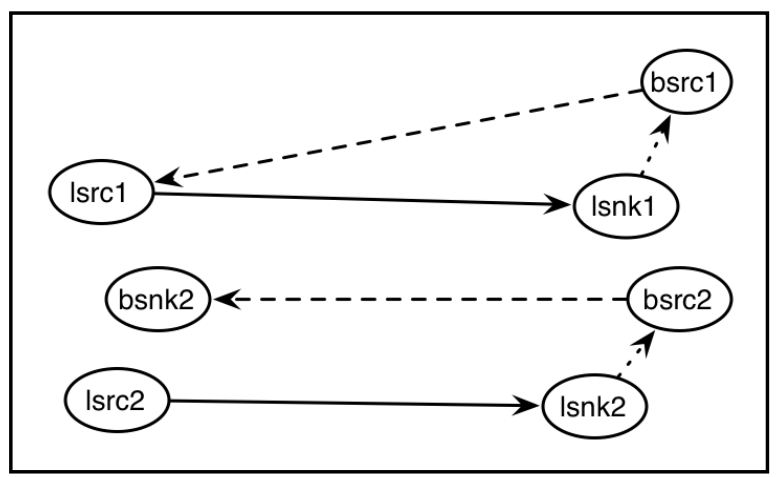

Fig. 1. Example logistics network with backhaul opportunities.

\section{BINARy INTEgER PROGRAMMING MODEL FOR BACKHAUL MATCHING PROBLEM}

Based on our problem scenario, each decision variable represents the matching decision for each possible pair of linehaul and backhaul shipments. As a result, it can be modeled as a binary variable. More specifically, the matching decision becomes 1 if a linehaul shipment can be matchedwith a particular backhaul shipment, otherwise 0 . Consequently, our backhaul matching problem can be modeled as a Binary Integer Programming (BIP) model. To facilitate our discussion, the variables and parameters involved in the model are presented in Table I.

TABLE I: RELATED VARIABLES AND MODEL PARAMETERS

\begin{tabular}{|c|c|}
\hline Variable/Parameter & Description \\
\hline$O_{L H}$ & The total numbers of linehaul shipments \\
\hline$O_{B H}$ & The total numbers of backhaul shipments \\
\hline$i$ & Index of linehaul shipment \\
\hline$j$ & Index of backhaul shipment \\
\hline$k$ & Type of truck ( 1 = flatbed trailer, $2=$ dump truck $)$ \\
\hline$R_{i}$ & $\begin{array}{l}\text { Truck type requirement for linehaul shipment } i \\
(1=\text { flatbed trailer, } 2=\text { dump truck) }\end{array}$ \\
\hline$R_{j}$ & $\begin{array}{l}\text { Truck type requirement for backhaul shipment } j \\
(1=\text { flatbed trailer, } 2=\text { dump truck) }\end{array}$ \\
\hline$x_{i j k}$ & $\begin{array}{l}\text { Matching decision for a pair of linehaul shipment } i \\
\text { and backhaul shipment jusing truck type } k \\
(0=\text { not matched, } 1=\text { matched })\end{array}$ \\
\hline$d_{i j}$ & $\begin{array}{l}\text { Deadhead distancebetween the linehaul shipment } i \\
\text { and the backhaul shipment } j\end{array}$ \\
\hline$D$ & $\begin{array}{l}\text { Maximum deadhead distance as allowed by } \\
\text { business policy }\end{array}$ \\
\hline$t_{L H i}$ & Delivery date of linehaul shipment $i$ \\
\hline$t_{B H j}$ & Pick-up date of backhaul shipment $j$ \\
\hline$T$ & $\begin{array}{l}\text { Maximum overhead time between linehaul delivery } \\
\text { and backhaul pick-up as allowed by business policy }\end{array}$ \\
\hline$C_{b k}$ & Base capacity of the truck type $k$ \\
\hline$C_{s k}$ & Spot capacity of the truck type $k$ \\
\hline$P_{i}$ & Product category of linehaul shipment $i$ \\
\hline$P_{j}$ & Product category of backhaul shipment $j$ \\
\hline
\end{tabular}

We aim to maximize the total number of backhaul matchings by considering all possible pairs of linehaul and backhaul shipments delivered by both flatbed and dump trucks. Accordingly, our objective function becomes:

$$
\operatorname{Max} \sum_{i} \sum_{j} \sum_{k} x_{i j k}
$$

While targeting the above objective function, our challenge is also to satisfy various operational and logical constraints, as depicted in the following constraint $(\mathrm{C} 1)$ to (C10). First, constraint (C1) states that each matching decision $x_{i j k}$ is represented by a binary decision variable. Constraint (C2) controls that the deadhead distance $d_{i j}$ of each matching pair cannot exceed thepredefined value $D$. Moreover, constraint (C3) regulates that the delay time between the linehaul delivery date, $t_{L H i}$, and the backhaul pick-up date, $t_{B H}$, is limited to the maximum overhead time $T$.

$$
x_{i j k=}\{0,1\}
$$

$$
\begin{aligned}
& x_{i j k} d_{i j} \leq D ; \forall i, j \text { for } k=1,2 \\
& x_{i j k}\left(t_{B H j}-t_{L H i}\right) \leq T ; \forall i, j \text { for } k=1,2
\end{aligned}
$$

For each matching, constraint (C4) and (C5) state that the truck type must conform to thetruck type requiredby both linehaul and backhaul products. Furthermore, constraint (C6) shows that for a truck type $k$, the number of matchings must 
not exceed its total number of base capacity, $C_{b k}$, and its spot capacity, $C_{s k}$.

$$
\begin{gathered}
\sum_{i} x_{i j k}=0 ; R_{i} \neq k \\
\sum_{j} x_{i j k}=0 ; R_{j} \\
\sum_{i} \sum_{j} x_{i j k} \leq C_{b k}+C_{s k} ; \text { for } k=1,2
\end{gathered}
$$

Constraint (C7) governs that any pair of linehaul and backhaul shipments with conflicting product categories shall not be matched. For example, trucks should not pick up foods after delivering construction materials.

$$
\begin{gathered}
\sum_{j} \sum_{k} x_{i j k}=0 ; \text { for all pair of linehaul } i \\
\text { and backhual } i \text { that } \\
P_{i} \text { and } P_{j} \text { confict }
\end{gathered}
$$

Lastly, constraint (C8), (C9), and (C10) ensure the validity of the model. The number of matchings must not surpass the minimum between the number of linehaul shipments and the quantity of backhaul shipments. Moreover, each of the linehaul and backhaul shipments must be matched only once.

$$
\begin{aligned}
& \sum_{i} \sum_{j} \sum_{k} x_{i j k} \leq \min \left[O_{L H}, O_{B H}\right] \\
& \sum_{j} \sum_{k} x_{i j k} \leq 1 \quad \forall i \\
& \sum_{i} \sum_{k} x_{i j k} \leq 1 \quad \forall j
\end{aligned}
$$

The developed BIP model is then implementedwithin an actual 3PL network in Thailand as presented in the next section.

\section{A CASE Study in a 3PL Network In Thailand}

The prototype software of our proposed BIP model was developed to provide suggestions on daily optimal backhaul matching plan for a giant 3PL network in Thailand. The transportation operations of the network involve a broad variety of products from a large customer portfolio such as construction materials, agricultural, pulp and paper, etc. For illustration, we conducted our study on the logistics network in the north-eastern region of Thailand.

To preserve data privacy, all the product names and transported volume shown here have been coded and transformed. However, the presented data still represent the realistic behavior of the input data and their corresponding results.

The logistics network with backhaul matching opportunity under study involves 5 and 15 categories of linehaul products and backhaul products respectively. A linehaul product is usually shipped from the linehaul source on day $t$, delivered to the linehaul sink on day $t+1$. Then, the truck picks up the backhaul product at its source on day $t+2$. The backhaul matching plan is an operational plan for daily use. The plan is

\begin{tabular}{|c|c|c|c|c|c|c|c|c|c|c|}
\hline \multirow[b]{2}{*}{ Product Category } & \multicolumn{5}{|c|}{ Linehaul } & \multicolumn{5}{|c|}{ Backhaul } \\
\hline & 1 & 2 & 3 & 4 & ... and so on & 1 & 2 & 3 & 4 & ... and so on \\
\hline Truck Type & 1 & 1 & 2 & 2 & ... and so on & 1 & 2 & 1 & 1 & ... and so on \\
\hline \multicolumn{11}{|l|}{ Volume (trips/day) } \\
\hline $6 / 3 / 2013$ & 129 & 6 & 12 & 6 & ... and so on & 16 & 0 & 18 & 0 & ... and so on \\
\hline $6 / 4 / 2013$ & 111 & 0 & 11 & 0 & ... and so on & 41 & 0 & 15 & 3 & ... and so on \\
\hline $6 / 5 / 2013$ & 122 & 3 & 10 & 10 & ... and so on & 21 & 0 & 17 & 0 & ... and so on \\
\hline ... and so on & & & & & & & & & & \\
\hline
\end{tabular}
created every morning before $10 \mathrm{a} . \mathrm{m}$. on estimate. Table II shows an example of order volume received per day. Due to

\begin{tabular}{|c|c|c|c|c|c|c|c|c|c|c|c|c|c|c|c|c|}
\hline & & \multicolumn{15}{|c|}{ Backhaul Categories } \\
\hline & & 1 & 2 & 3 & 4 & 5 & 6 & 7 & 8 & 9 & 10 & 11 & 12 & 13 & 14 & 15 \\
\hline \multirow{5}{*}{ 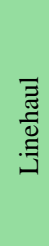 } & 1 & & & & & & & & & & & & & & & \\
\hline & 2 & & & & & & & & & & & & & & & \\
\hline & 3 & $\mathrm{X}$ & $\mathrm{X}$ & $\mathrm{X}$ & $\mathrm{X}$ & $\mathrm{X}$ & & $\mathrm{X}$ & $\mathrm{X}$ & $\mathrm{X}$ & $\mathrm{X}$ & $\mathrm{X}$ & $\mathrm{X}$ & $\mathrm{X}$ & $\mathrm{X}$ & $\mathrm{X}$ \\
\hline & 4 & $\mathrm{X}$ & $\mathrm{X}$ & $\mathrm{X}$ & $\mathrm{X}$ & $\mathrm{X}$ & $\mathrm{X}$ & & & $\mathrm{X}$ & $\mathrm{X}$ & $\mathrm{X}$ & $\mathrm{X}$ & $\mathrm{X}$ & $\mathrm{X}$ & $\mathrm{X}$ \\
\hline & 5 & & & & & & & & & & & & & & & \\
\hline
\end{tabular}
the limited space, only a portion of the dataset is displayed here. As an example, on June $3^{\text {rd }} 2013,129$ trucks of type 1 are required for delivering linehaul products of type 1. On the same day, 16 trucks of the same type are also needed to pick up backhaul products of type 1 .

TABLE II: EXAMPLE OF INPUT DATA, DAILY VOLUME ORDER

TABLE III: CONFLICT BETWEEN PRODUCT CATEGORIES

The information on the conflicts among the product categories is outlined in Table III. Each pair of the conflicting categories is marked with X. From Table III, linehaul category 3 shall be matched with backhaul category 6 only, whereas linehaul category 4 can be paired with backhaul category 7 and 8 .
Our BIP model was implemented in Java and solved using CPLEX optimizer V.12.4. An experiment was conducted for one month, using maximum deadhead distance $D=150 \mathrm{k} . \mathrm{m}$., overhead time $\operatorname{limit} T=2$, and whenbase capacity of the truck type $C_{b k}$ andspot truck capacity $C_{s k}$ are very large. 


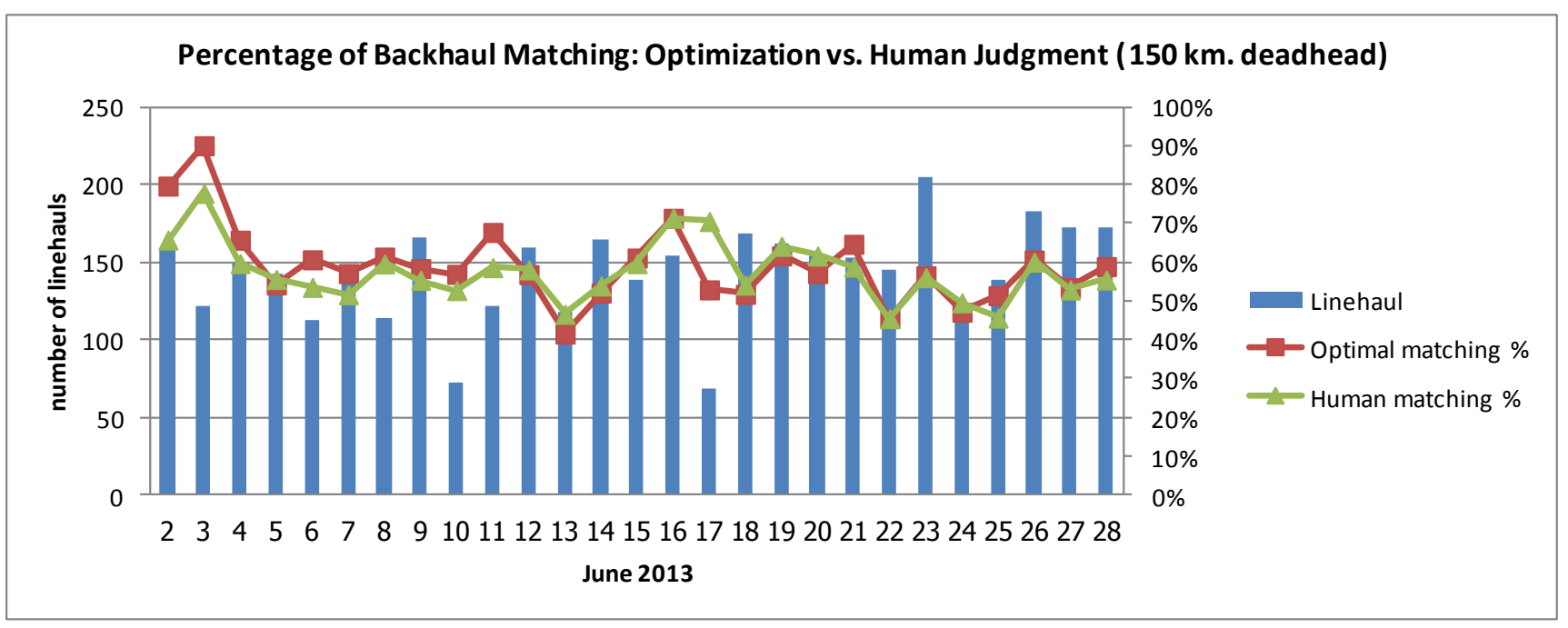

Fig. 2. Result comparison between optimized matching and manual matching.

\section{EXPERIMENTAL RESULTS AND DISCUSSION}

The result of our one-month experiment on backhaul matching operations is presented in Fig. 2. The bars in Fig. 2 show the volume of linehaul shipments on each particular day as numerated on the left y-axis. For instance, the linehaul volume on June $2^{\text {nd }}$ is slightly above 150 shipments; whereas, there are over 200 linehaul shipments on June $23^{\text {rd }}$.

The line graph on the same figure provides the comparison between the percentage of backhaul matching obtained from our optimization method and the manual operation based on human's judgment. In addition to avoiding labor-intensive time-consuming process, our optimization method gives the optimal objective value of approximately $59 \%$, whereas the manual operation produced a slightly inferior value at about $57 \%$.

Nevertheless, if we examine the matching outcome for each particular day; for instance on day $17^{\text {th }}$, it can be observed that the manual method sometimes provides higher level of matchingthan that from ouroptimization method.Further investigation revealed that there exist manual matchings that violates the limited deadhead constraints of $150 \mathrm{~km}$. For example, on day $17^{\text {th }}$, the linehaul product category 3 and backhaul product category 6 generates deadhead distance of $290 \mathrm{~km}$. This occurrence reflects additional advantages of our optimization method which avoids human errors and always maintains all of the business's constraint requirements.

Even though our proposed matching optimization solution has high potential for fortifying the backhaul matching operations in the logistics network under study, there is still room for further improvement to make the approach more practical. First of all, by working closely with operational staffs, the model could be modified to be more flexible and toaccommodate special operational requirements of the network such as exceptional matching pairs that should be allowed to have more relaxing constraints. Second, the proportion of the job assignment to the outsourced carriers, also known as the carrier quota, can be taken into account when generating the matching plan. Lastly, a proper software GUI is also needed to be develop.

\section{CONCLUSION}

This paper emphasizes backhaul matching optimization which serves as a great avenue for cost saving in transportation, particularly, in the operations of 3PL networks with a variety and large portion of products and customer portfolios. To obtain an optimal matching plan, a BIP model is developed with decision variables depicting whether each possible pair of linehaul and backhaul shipments should be matched. An experiment was conducted in a large 3PL network to validate the result from our model. Our preliminary study indicates that the proposed BIP model provides advantages over the traditional manual operations in several aspects and offers an opportunity for backhaul matching improvement. Moreover, it can be further enhanced to include additional constraints such ascarrier quota and to accommodate some exceptional user requirements.

\section{REFERENCES}

[1] Establish Supply Chain Management Consultants, Logistics Cost and Service 2012, Establish Davis Logistics Cost and Service Database, September 2013.

[2] Office of the National Economic and Social Development Board, Thailand's Logistics Report 2011, February 2012.

[3] M. Goetschalckx and C. Jacobs-Blecha, "The Vehicle Routing Problem with Backhauls," European Journal of Operational Research, vol. 42, no. 1, pp. 39-51, September 1989.

[4] J. Brandao, "A New Tabu Search Algorithm for the Vehicle Routing Problem with Backhauls," European Journal of Operational Research, vol. 173, no. 2, pp. 540-555, September 2006.

[5] N. Wassan, "Reactive Tabu Adaptive Memory Programming Search for the Vehicle Routing Problem with Backhauls," Journal of the Operational Research Society, vol. 58, no. 12, pp. 1630-1641, December 2007.

[6] W. NurfahizulIfwah. W. M. Shaiful, M. Z. Shamsunarnie, Z. M. Zainuddin, and M. Fuad, "Genetic Algorithm for Vehicle Routing Problem with Backhauls," Journal of Science and Technology, vol. 4, no. 1, pp. 9-16, June 2012.

[7] Y. Gajpal and P. L. Abad, "Multi-Ant Colony System (MACS) for a Vehicle Routing Problem with Backhauls," European Journal of Operational Research, vol. 196, no. 1, pp. 102-117, July 2009.

[8] E. E. Zachariadis and C. T. Kiranoudis, "An Effective Local Search Approach for the Vehicle Routing Problem with Backhauls," Expert Systems with Applications, vol. 39, no. 3, pp. 3174-3184, February 2012.

[9] D. Carlsson and M. Ronnqvist, "Backhauling in Forest Transportation: Models, Methods, and Practical Usage," Canadian Journal of Forest Research, vol. 37, no. 12, pp. 2612 - 2623, May 2007.

[10] W. Chen, L. Pi, L. Shi, B. Thomas, E. Wikum, G. Jarosinski, P. Sun, and T. Gifford, "Data Mining-Based Decision System Framework for 
Local Pickup and Delivery Problem," presented at INFORMS Annual Meeting, November7, 2007.

[11] J. Muckell, Q. Cao, P. Mackenzie, D. Messier, and J. Salvo, "Toward an Intelligent Brokerage Platform Mining Backhaul Opportunities in Telematics Data," Transportation Research Record: Journal of the Transportation Research Board, vol. 2097, pp. 1-8, September 2009.

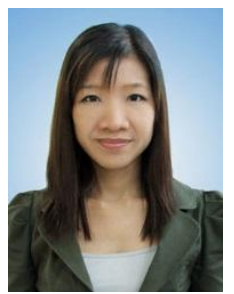

Machigar Ongtang received her Ph.D. in computer science and engineering from Pennsylvania State University in 2010, MSc in information technology for Manufacture from University of Warwick, UK in 2004.

Currently, she is an assistant professor at Faculty of Information Technology, Dhurakij Pundit University, Thailand. Her research interests include system engineering, software engineering, computer security, telecommunication security, and mobile technology.

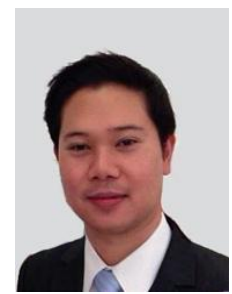

Satama Sirivunnabood completed his $\mathrm{Ph} . \mathrm{D}$. in industrial engineering and operations research from Pennsylvania State University in 2010, MSc in advanced manufacturing technology and systems management, University of Manchester, UK in 2004.

$\mathrm{He}$ is currently a supply chain analyst and also has appointment with Department of Industrial Engineering, King Mongkut's Institute of Technology Ladkrabang, Thailand. His research lies in the area of supply chain optimization, operations research, and IT in Supply Chain. 